$17^{\circ}$ ERGODESIGN

\& USIHC 2019

PUC-Rio, 11 a 13 de dezembro

Rio de Janeiro, RJ, Brasil $17^{\circ}$ Ergodesign - Congresso Internacional de Ergonomia e Usabilidade de Interfaces Humano Tecnológica: Produto, Informações Ambientes Construídos e Transporte

$17^{\circ}$ USIHC - Congresso Internacional de Ergonomia e Usabilidade

de Interfaces Humano Computador

\title{
Análise Ergonômica do Processo de Produção Artesanal: uma revisão sistemática da literatura
}

\author{
Ergonomic Analysis of the Craft Production Process: a systematic review of \\ the literature
}

\author{
ARAÚJO, Layane Nascimento de \\ Universidade Federal de Pernambuco, Mestranda em Design \\ layane.n.araujo@gmail.com \\ VILLAROUCO, Vilma \\ PPGDesign-UFPE; PPErgo-UFPE; PPGAU+D-UFC; Dra. Eng. \\ vvillarouco@gmail.com
}

\author{
ALBUQUERQUE, Sheila Rodrigues de \\ Universidade Federal de Pernambuco, Mestranda em Design \\ sheilaarq1@gmail.com
}

\section{RESUMO}

Este artigo tem como propósito revisar, na literatura, estudos que abordem a Análise Ergonômica em Processos de Produção Artesanais. A pesquisa foi realizada na base de Periódico da CAPES. O objetivo da busca foi o de identificar metodologias, ferramentas e métodos utilizados nesses estudos. Como descritores foram utilizadas as palavras-chaves na língua portuguesa: "Artesanato, ergonomia, Análise ergonômica, processo de produção e processo de produção artesanal" e na língua inglesa: "Craft, ergonomics, Ergonomic analysis, production process, Craft production process". A busca focou artigos dos últimos 5 anos e idiomas inglês e português. Foram encontrados 40.819 artigos, após tal resultado foi adicionado o filtro "revisado por pares", dos quais foram analisados os 100 primeiros das 12 categorias buscadas, ou seja, 784 artigos foram analisados por títulos, os repetidos foram excluídos, resultando assim, em 15 artigos que seriam analisados pelo resumo. Após a leitura dos resumos, restaram 10 artigos, que foram divididos em grupos de 1 à 3 dependendo do grau de relevância para a pesquisa. As análises ergonômicas corresponderam em sua maioria a avaliações posturais, destacando as lesões ocasionadas por tais, bem como a ocorrência de Lesão por Esforço Repetitivo (LER). Em alguns casos o ambiente de trabalho também foi estudado.

Revisão sistemática, Análise ergonômica, Processo de produção, Artesanato.

\begin{abstract}
This article aims to review, in literature, studies that address to Ergonomic Analysis in Craft Production Processes. The research was conducted based on CAPES Journal. The objective of the search was to identify methodologies, tools and methods used in these studies. The keywords used in the Portuguese language were: "Artesanato, ergonomia, Análise ergonômica, processo de produção e processo de
\end{abstract}


produção artesanal" and in English: "Craft, Ergonomics, Ergonomic Analysis, Production Process, Craft Production Process". The search focused on articles from the last 5 years in English and Portuguese languages. 40,819 articles were found, after this result the "peer reviewed" filter was added, from which we analyzed the first 100 of the 12 searched categories, that is, 784 articles were analyzed by titles, the repeated ones were excluded, resulting in 15 articles that would be analyzed by the abstract. After reading the abstracts, 10 articles remained, which were divided into groups from 1 to 3 depending on the degree of relevance to the research. The ergonomic analyzes corresponded mostly to postural evaluations, highlighting the injuries caused by such, as well as the occurrence of Repetitive Strain Injury (RSI). In some cases the work environment has also been studied.

Systematic Review, Ergonomic Analysis, Production Process, Craft.

\section{INTRODUÇÃO}

Caracterizado pela técnica manual, o artesanato compreende tanto valores simbólicos, quanto culturais e sociais. Várias são as tipologias de artesanato identificadas ao redor do mundo, que variam de matéria-prima para o seu processo produtivo, desde o barro, madeira, fios, pedra, ferro, couro, fibras e até mesmo papel.

Por suas características, por vezes, de alta informalidade, depreende-se a necessidade de estudar esse setor produtivo sob o enfoque da ergonomia, sendo este o foco deste presente trabalho, que visa identificar como tal enfoque tem sido adotado nos estudos realizados e publicados.

Para o desenvolvimento desta pesquisa foi realizado um amplo levantamento bibliográfico, por meio de uma revisão sistemática da temática abordada, análise ergonômica do processo de produção artesanal. Foram avaliados estudos em bases de dados pertencentes ao portal Periódicos CAPES - Coordenação de Aperfeiçoamento de Pessoal de Nível Superior, com o intuito de identificar na literatura trabalhos, desde artigos, até dissertações e teses, que trabalhassem essa temática, e os métodos e ferramentas que foram utilizados nos mesmos para a execução da análise ergonômica. Bem como, as problemáticas levantadas e os resultados obtidos.

Esta pesquisa foi desenvolvida a partir do método PRISMA - Preferred Reporting Items for Systematic Reviews and Meta-Analyses, e se configura em um estudo analítico uma vez que envolve uma avaliação mais aprofundada das informações que forem coletadas, na tentativa de explicar os artigos selecionados.

\section{ARTESANATO E ERGONOMIA}

O artesanato é uma atividade passada de geração em geração e muitas vezes funciona como principal fonte de renda das famílias que o fazem. O mesmo abrange tanto âmbitos culturais, quanto sociais, e se enquadra como uma prática vernacular, já que para sua prática são empregados materiais e recursos do próprio ambiente, reverberando as características locais e regionais. Pode-se dizer que o "fazer manual" rebate o sistema de produção globalizado e industrializado, promovendo a manutenção da cultura e da identidade local.

Segundo o Programa Nacional do Artesão Brasileiro (PAB), encaixa-se como artesão aqueles que utilizam matéria-prima natural, que podem ser de origem mineral, vegetal ou animal, e processo de produção que pode ser artesanal ou por meio de reciclagem e reaproveitamento, para a criação de artefatos, tais como joalherias, cerâmicas, movelaria, tapeçarias, sapataria, rendas e bordados, por exemplo. Desse modo é emitida no Brasil, desde 2012, pela Secretaria da Micro e Pequena Empresa (SMPE) com o apoio do Programa Nacional do Artesão (PAB), a 


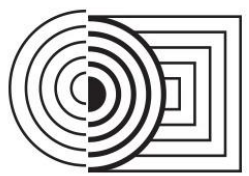

\section{$17^{\circ}$ ERGODESIGN \& USIHC 2019 \\ PUC-Rio, 11 a 13 de dezembro \\ Rio de Janeiro, RJ, Brasil}

$17^{\circ}$ Ergodesign - Congresso Internacional de Ergonomia e Usabilidade de Interfaces Humano Tecnológica: Produto, Informações Ambientes Construídos e Transporte

$17^{\circ}$ USIHC - Congresso Internacional de Ergonomia e Usabilidade de Interfaces Humano Computador

Carteira Nacional do Artesão e a do Trabalhador Manual como meio de identificação nacional para essa classe de trabalhadores.

Segundo Duarte et al (2015) estas comunidades apresentam, além do conhecimento e sistemas compatíveis com o ambiente (memória étnica), técnicas manuais passadas entre gerações (memória técnica), ou seja, detém conhecimentos específicos sobre manejo, técnicas de transformação de matéria-prima e dinâmica do ecossistema ao qual está inserida. $E$ ainda, a produção artesanal tem forte ligação com o conhecimento tradicional e a tradição oral (DUARTE et al, 2015).

Entretanto, a profissão do artesão também oferece seus riscos e quando se trata de melhorias no processo produtivo, práticas artesanais acabam sendo esquecidas, em virtude de que muitas vezes não se levantam questionamentos com relação às inadequações ergonômicas dos postos de trabalhos dos artesãos.

Para a ABERGO (2018), é necessário que o ergonomista possua uma abordagem holística entendendo o usuário, seu contexto e as demais variantes do processo, tanto em seus aspectos físicos e cognitivos, como sociais, organizacionais e ambientais. E tal abordagem se faz obrigatória para a identificação de problemáticas no processo produtivo artesanal. Tal proposição mostra que a ergonomia visa orientar o bem-estar do indivíduo, garantindo sua segurança, contudo sem deixar de contemplar a sua produtividade e a sua qualidade de trabalho.

\section{MÉTODO}

Como procedimento metodológico utilizado para coleta de dados, esta pesquisa utiliza a Revisão Sistemática da Literatura (RSL), em virtude de que a mesma visa investigar cientificamente, para aumentar o potencial da busca e reunir estudos relevantes, sobre determinado conteúdo. Para Cordeiro et al (2007):

A revisão sistemática é um tipo de investigação científica que tem por objetivo reunir, avaliar criticamente e conduzir uma síntese dos resultados de múltiplos estudos primários. Ela também objetiva responder a uma pergunta claramente formulada, utilizando métodos sistemáticos e explícitos para identificar, selecionar e avaliar as pesquisas relevantes, coletar e analisar dados de estudos incluídos na revisão (CORDEIRO ET AL, 2007).

Desse modo, para a execução dessa pesquisa realizou-se uma revisão sistemática do tipo qualitativa e para tanto foi utilizado o método PRISMA. Segundo a Secretaria de Ciência, Tecnologia e Insumos Estratégicos Departamento de Ciência e Tecnologia do Ministério da Saúde, Brasil (2012), o PRISMA surgiu de uma revisão e atualização do QUOROM (Quality Of Reporting Of Metaanalyses), e a principal razão da mudança de nome foi devido à necessidade de se diferenciar Revisão Sistemática de Metanálise. Ainda com base no Ministério da Saúde (2012), o PRISMA (Preferred Reporting Items for Systematic Reviews and Meta-Analyses) é uma diretriz que tem como objetivo ajudar autores a melhorarem a qualidade do relato dos dados da RS (Revisão Sistemática) e Metanálise.

\subsection{Revisão Sistemática}

Para a realização da Revisão Sistemática e utilização do método PRISMA, a base consultada foi a de periódicos CAPES, selecionada devido a inclusão de um número considerável de periódicos na realização de suas buscas: Scopus (Elsevier), SciELO (CrossRef), 
MEDLINE/PubMed (NLM), entre outros, bem como sua Base de Teses e Dissertações. O objetivo geral da busca foi o de identificar metodologias, ferramentas e métodos utilizados na análise ergonômica no segmento.

Buscou-se artigos, teses e dissertações e foram utilizadas como descritores as palavras-chaves na língua portuguesa: "Artesanato, ergonomia, Análise ergonômica, processo de produção e processo de produção artesanal" e na língua inglesa: "Craft, ergonomics, Ergonomic analysis, production process, Craft production process", combinadas entre si, ou de forma individual a fim de abranger o maior número de estudos sobre a temática. A primeira busca focou apenas artigos e ocorreu entre os meses de outubro e novembro de 2018 e teve como critérios de Inclusão e Exclusão, a Figura 1. Já a segunda busca ocorrei entre os meses de março e abril de 2019 e teve como critérios de Inclusão e exclusão a Figura 2.

Figura 1. Critérios de Inclusão e Exclusão da busca dentro do Portal de Periódico CAPES

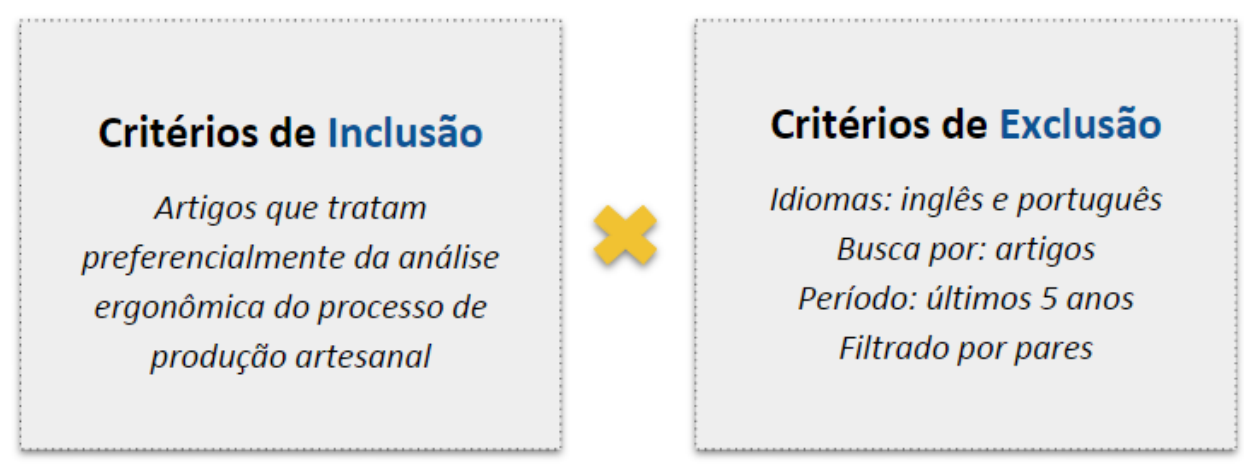

Fonte: autoras (2019)

Figura 2. Critérios de Inclusão e Exclusão da busca dentro do Portal de Teses e Dissertações da CAPES

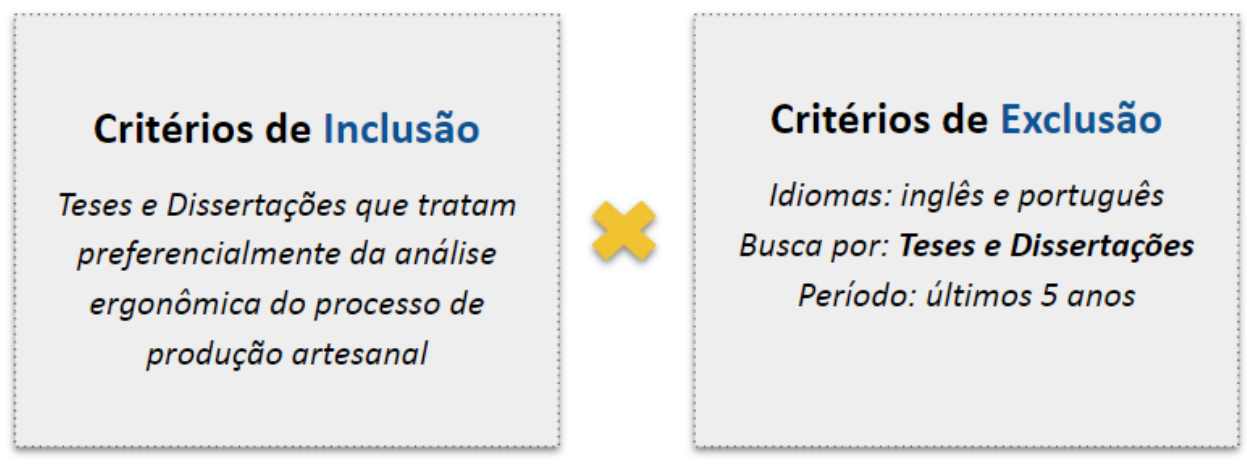

Fonte: autoras (2019)

A ordem do passo-à-passo da execução da Revisão Sistemática e do método PRISMA, para melhor compreensão, pode ser conferida na Figura 3 a seguir. 


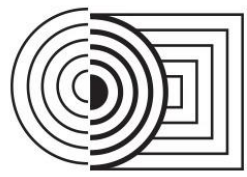

$17^{\circ}$ ERGODESIGN \& USIHC 2019

PUC-Rio, 11 a 13 de dezembro

Rio de Janeiro, RJ, Brasil $17^{\circ}$ Ergodesign - Congresso Internacional de Ergonomia e Usabilidade de Interfaces Humano Tecnológica: Produto, Informações Ambientes Construídos e Transporte

$17^{\circ}$ USIHC - Congresso Internacional de Ergonomia e Usabilidade de Interfaces Humano Computador

Figura 3. Passo-à-passo da Revisão Sistemática

Busca Geral nos periódicos CAPES e no Portal CAPES de Teses e Dissertações

Filtro por Título

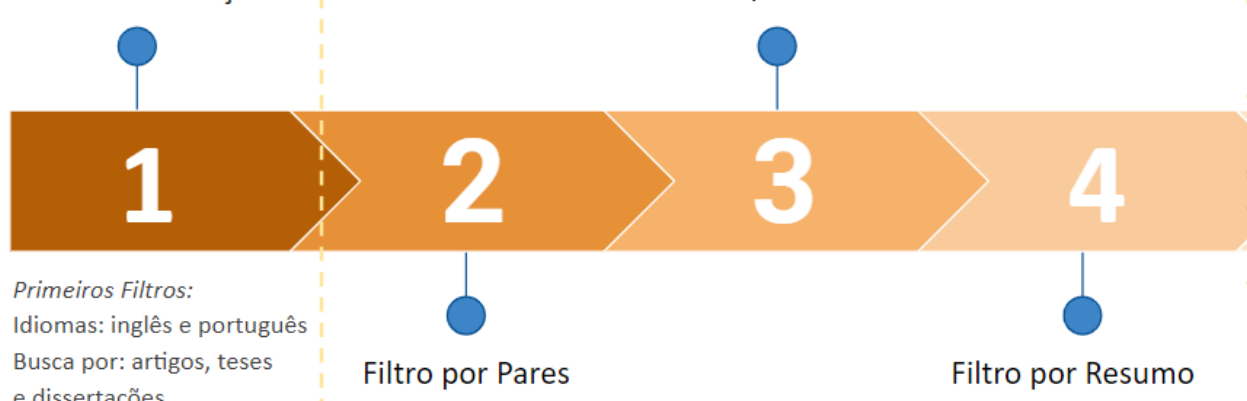

e dissertações

Período: últimos 5 anos

Fonte: autoras (2019)

Nesse caso as etapas consistem, primeiramente, na busca geral por artigos, no Portal de Períodos da CAPES, e trabalhos, no Portal de Teses e Dissertações, apenas nos idiomas inglês e português, e nos últimos 5 anos (2013-2018). Após tais filtros, outro filtro foi adicionado na busca por artigos a fim de que essa fosse mais refinada para Periódicos revisados por pares, isto é, apenas para artigos avaliados por ao menos dois avaliadores. Em seguida, é feita uma busca nos títulos dos artigos e dos trabalhos (teses e dissertações) a fim de analisar e separar os de maiores relevâncias, para que posteriormente possa ser feita uma busca nos seus Resumos a fim de especificar os que deveriam ser analisados por completo.

\subsubsection{Busca por Artigos}

Nesta primeira fase do estudo, a busca concentrou-se somente em artigos do Portal de Periódicos Capes, e as suas etapas serão descritas a seguir.

\section{Etapa 1 e 2}

A primeira busca, retornou um total geral de $\mathbf{4 0 . 8 1 9}$ artigos, já filtrados por idioma e por ano últimos 5 anos. Desses, após a seleção do filtro: periódicos revisados por pares, houve um decréscimo de 3.638 artigos, resultando em $\mathbf{3 7 . 1 8 1}$ artigos encontrados. As combinações das palavras-chaves utilizadas nas buscas filtradas por pares podem ser verificadas nas tabelas 1 , em inglês, e 2, em português, respectivamente, a seguir:

Tabela 1. Buscas nos periódicos CAPES - combinações em inglês

\begin{tabular}{c|c}
\hline $\begin{array}{l}\text { Categoria da busca: Qualquer - Qualquer } \\
\text { Palavra-chave / Booleano / Palavra-chave }\end{array}$ & Retorno (em no de artigos filtrados por pares) \\
\hline Craft and Ergonomics & 381 \\
\hline Craft and Ergonomic Analysis & 168 \\
\hline Craft and Production Process & 17.787 \\
\hline Craft Production Process and Ergonomics & 158 \\
\hline
\end{tabular}




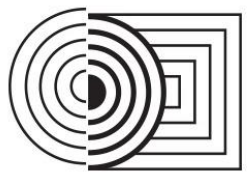

$17^{\circ}$ ERGODESIGN \& USIHC 2019

PUC-Rio, 11 a 13 de dezembro

Rio de Janeiro, RJ, Brasil $17^{\circ}$ Ergodesign - Congresso Internacional de Ergonomia e Usabilidade de Interfaces Humano Tecnológica: Produto, Informações Ambientes Construídos e Transporte

$17^{\circ}$ USIHC - Congresso Internacional de Ergonomia e Usabilidade de Interfaces Humano Computador

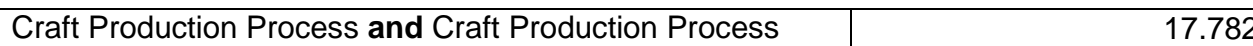
Craft Production Process and Ergonomic Analysis 76

Fonte: autoras (2019)

Tabela 2. Buscas nos periódicos CAPES - combinações em português

\begin{tabular}{c|c}
\hline $\begin{array}{c}\text { Categoria da busca: Qualquer - Qualquer } \\
\text { Palavra-chave / Booleano / Palavra-chave }\end{array}$ & Retorno (em no de artigos filtrados por pares) \\
\hline Artesanato and Ergonomia & 3 \\
\hline Artesanato and Análise Ergonômica & 1 \\
\hline Processo de produção artesanal and Análise Ergonômica & 0 \\
\hline Processo de produção artesanal and Processo de produção \\
artesanal & 121 \\
\hline Processo de produção artesanal and Ergonomia & 6 \\
\hline Processo de produção and Artesanato & 98 \\
\hline
\end{tabular}

Fonte: autoras (2019)

Para as buscas das palavras-chaves o Portal de periódicos CAPES disponibiliza as categorias: Qualquer, Busca no Título, Busca como autor e Busca no Assunto. Inicialmente foram utilizadas as categorias: Busca no Título e Busca no Assunto, com os booleanos and (e) e or (ou). No entanto verificou-se que tal especificidade reduziu drasticamente o número de artigos encontrados. Logo para aumentar a gama de artigos a serem encontrados, foram utilizadas as categorias de busca Qualquer - Qualquer, como pode ser observado nas tabelas 1 e 2, e o booleano selecionado para tanto foi o booleano and, uma vez que com o uso do or, os resultados das buscas não eram direcionados para a pesquisa em si.

As tabelas 1 e 2, ainda, mostram que as publicações em inglês apresentam um total de $\mathbf{3 6 . 9 5 2}$ artigos encontrados, enquanto as buscas em português apresentam um total de apenas 229 artigos.

\section{Etapa 3 e 4}

$\mathrm{Na}$ etapa 3 foram analisados à priori, por Título, os 100 primeiros artigos mais relevantes de cada um dos 12 grupos e combinações das palavras-chaves. E, após exclusão de artigos repetidos, obteve-se um número total de 15 artigos. Em seguida, na etapa 4, foram lidos os resumos desses, restando $\mathbf{1 0}$ artigos que foram analisados em leitura completa. A Figura 4 ilustra estas etapas. 
$17^{\circ}$ ERGODESIGN \& USIHC 2019

PUC-Rio, 11 a 13 de dezembro

Rio de Janeiro, RJ, Brasil $17^{\circ}$ Ergodesign - Congresso Internacional de Ergonomia e Usabilidade de Interfaces Humano Tecnológica: Produto, Informações Ambientes Construídos e Transporte

$17^{\circ}$ USIHC - Congresso Internacional de Ergonomia e Usabilidade

de Interfaces Humano Computador

Figura 4. Etapas 3 e 4 do processo de RSL - Artigos

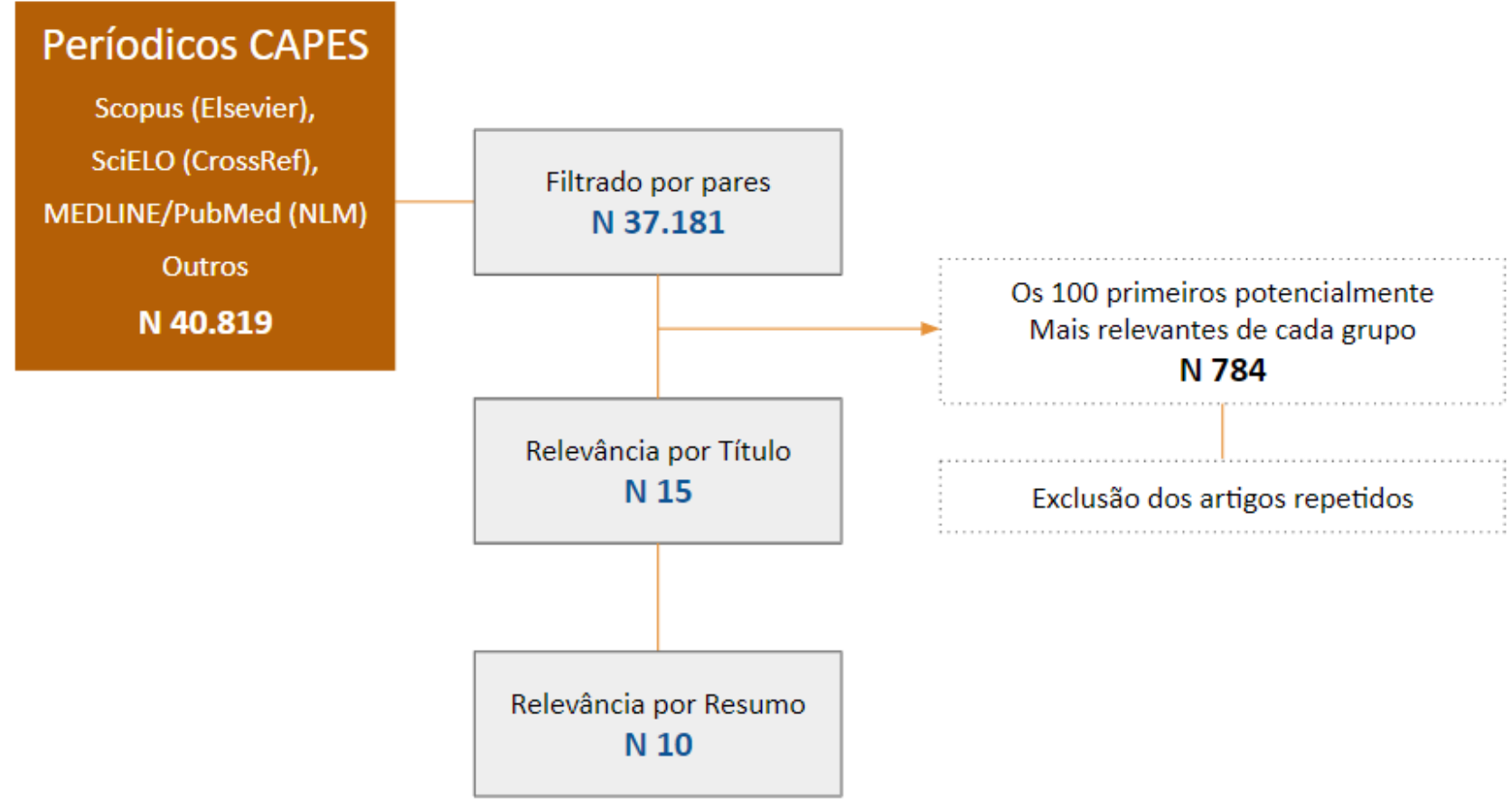

Fonte: autoras (2019)

Com base no objetivo geral da pesquisa, foram poucos os artigos encontrados com o foco principal do estudo, de identificar metodologias, ferramentas e métodos utilizados para análise ergonômica de processos de produções artesanais. Diante disso, a busca foi abrangida para estudos que contribuíssem para processos de produção artesanais contemporâneos, resultando no total de 10 artigos a serem analisados na etapa 5, seguinte.

\section{Etapa 5}

Nesta etapa, foram lidos completamente os 10 artigos selecionados por resumo. Inicialmente foi realizada uma leitura flutuante para estabelecer primeiro contato com os documentos submetidos à análise e reconhecimento das temáticas. Em um segundo momento, foi realizada exploração do material a partir da leitura exaustiva dos textos, que permitiu dividir os artigos em grupos.

Tais grupos variam de 1 a 3, de acordo com a relevância do contexto do artigo para a temática abordada - análise ergonômica do processo de produção artesanal (ver Figura 5), e suas informações foram organizadas em colunas na planilha Excel. 
$17^{\circ}$ ERGODESIGN \& USIHC 2019

PUC-Rio, 11 a 13 de dezembro Rio de Janeiro, RJ, Brasil $17^{\circ}$ Ergodesign - Congresso Internacional de Ergonomia e Usabilidade de Interfaces Humano Tecnológica: Produto, Informações Ambientes Construídos e Transporte

$17^{\circ}$ USIHC - Congresso Internacional de Ergonomia e Usabilidade de Interfaces Humano Computador

Figura 5. Grupos dos artigos analisados

Grupos:

Relevância Total

4 artigos
2

Relevância considerável 2 artigos

Fonte: autoras (2019)
3 Pouca Relevância

4 artigos

Sendo o grupo 1 para total relevância e presença da análise ergonômica no processo de produção artesanal; 2 para relevância considerável, isto é, há a abordagem ergonômica, porém, a temática não é tão pertinente à pesquisa; E 3 para pouca relevância e a não presença da análise ergonômica nos processos de produção artesanais.

\subsubsection{Busca por Teses e Dissertações}

Já no segundo momento, a busca focou no Portal de Teses e Dissertações da CAPES, e suas etapas serão descritas em seguida.

\section{Etapas 1 e 2}

Para a segunda busca, as combinações das palavras-chaves utilizadas podem ser verificadas nas tabelas 3 e 4, mais adiante. No entanto, embora o Portal de Teses e Dissertações não possua o filtro pelo idioma, uma vez que se concentra no Brasil e consequentemente no idioma português, a busca foi feita em ambos os termos em inglês e português, já que é obrigatório o uso de um resumo em inglês e em português. O único filtro utilizado foi de trabalhos realizados nos últimos 5 anos.

Tabela 3. Buscas no Portal de Teses e Dissertações - CAPES - Termos em inglês

\begin{tabular}{c|c}
\hline $\begin{array}{c}\text { Categoria da busca: Qualquer - Qualquer } \\
\text { Palavra-chave / Booleano / Palavra-chave }\end{array}$ & Retorno (em no de artigos filtrados por pares) \\
\hline Craft and Ergonomics & 365758 \\
\hline Craft and Ergonomic Analysis & 366044 \\
\hline Craft and Production Process & 366402 \\
\hline Craft Production Process and Ergonomics & 366404 \\
\hline Craft Production Process and Craft Production Process & 366402 \\
\hline Craft Production Process and Ergonomic Analysis & 366658 \\
\hline
\end{tabular}

Fonte: autoras (2019)

Tabela 4. Buscas no Portal de Teses e Dissertações - CAPES - combinações em português

\begin{tabular}{c|c}
\hline $\begin{array}{l}\text { Categoria da busca: Qualquer - Qualquer } \\
\text { Palavra-chave / Booleano / Palavra-chave }\end{array}$ & Retorno (em no de artigos filtrados por pares) \\
\hline Artesanato e Ergonomia & 365911 \\
\hline Artesanato e Análise Ergonômica & 402945 \\
\hline Processo de produção artesanal e Análise Ergonômica & 403391 \\
\hline
\end{tabular}




\begin{tabular}{c|c}
\hline $\begin{array}{c}\text { Processo de produção artesanal e Processo de produção } \\
\text { artesanal }\end{array}$ & 403391 \\
\hline Processo de produção artesanal e Ergonomia & 403391 \\
\hline Processo de produção e Artesanato & 403391 \\
\hline
\end{tabular}

Fonte: autoras (2019)

Foi observado que a busca retornou um total geral de 2.197.668 artigos, para os termos em inglês, e 2.382.420 artigos para os termos português.

\section{Etapa 3 e 4}

Pode-se verificar na etapa 3 a ocorrência de repetições nos trabalhos buscados. Dessa maneira, foram analisados por Título, os 100 primeiros artigos mais relevantes de cada um dos 12 grupos e combinações das palavras-chaves. Excluindo os trabalhos repetidos, chegou-se ao número de 18 trabalhos, sendo 13 Dissertações e 5 Teses. Na etapa 4, esses trabalhos foram analisados pelo resumo, e ao final restou apenas 1 trabalho, do tipo tese. O esquema do processo pode ser conferido na Figura 6.

Figura 6. Etapas 3 e 4 do processo de RSL - Dissertações e Teses

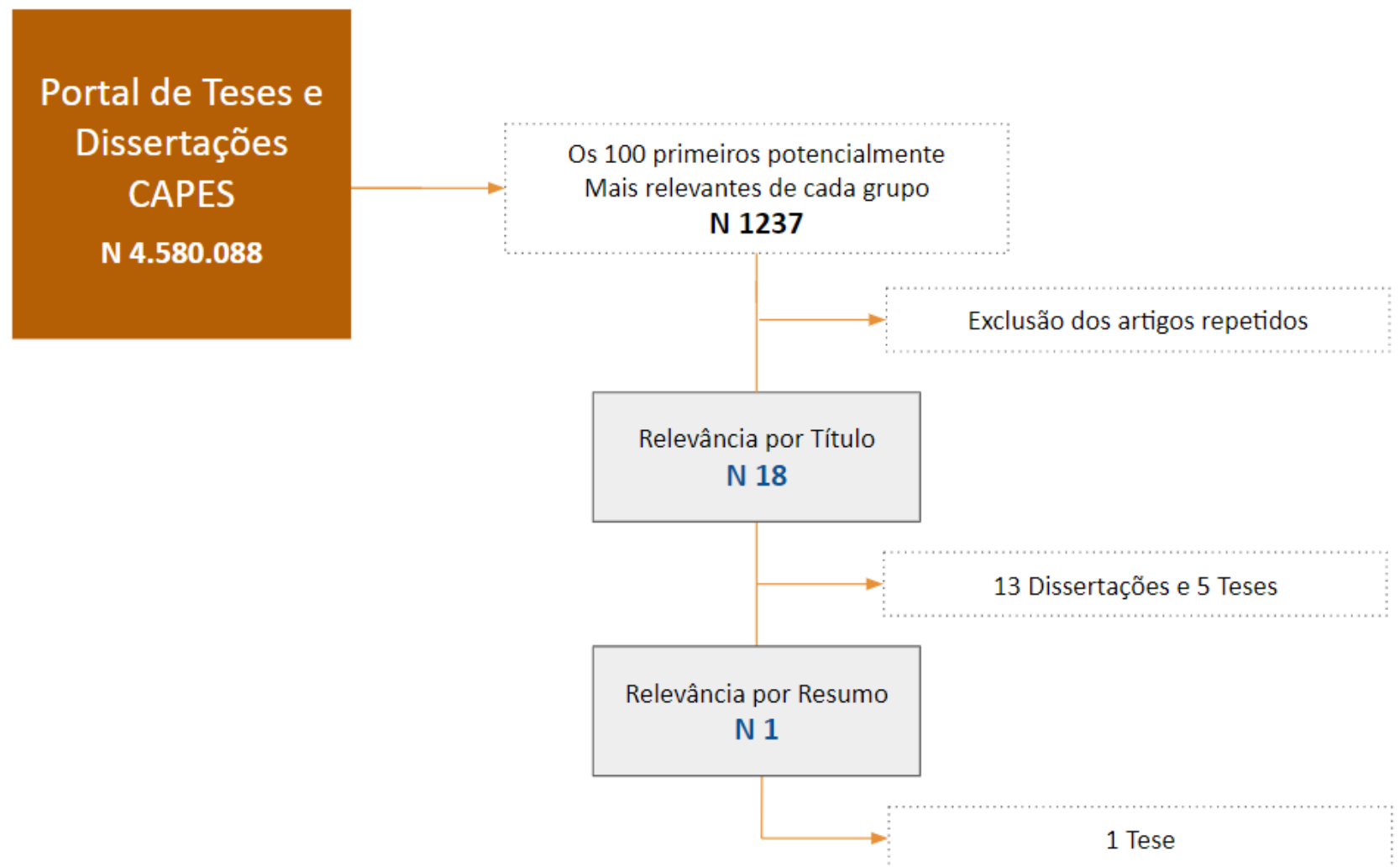

Fonte: autoras (2019)

A pesquisa manteve a diretriz de identificar trabalhos que realizassem análise ergonômica em processos de produções artesanais, entretanto não houve nenhum trabalho encontrado com 
esse foco. Dessa forma, a busca foi abrangida para estudos que contribuíssem para processos de produção artesanais, resultando no total de 1 tese a ser analisada.

\section{Etapa 5}

Após a leitura da tese, a mesma pode ser alocada na categoria 3 descrita no tópico anterior, como sendo de Pouca Relevância. Uma vez que não apresenta análise ergonômica de fato, mas sim um modelo de intervenção de design para ambientes artesanais. Logo atualizando o esquema dos grupos analisados, temos a Figura 7 a seguir.

Figura 7. Grupos dos trabalhos analisados e suas quantidades

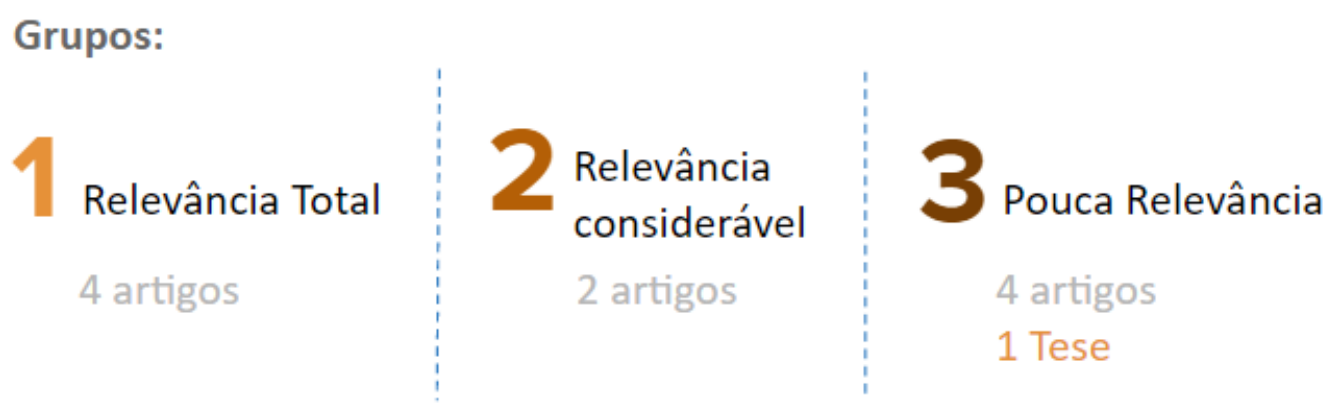

Fonte: autoras (2019)

A seguir serão apresentados e discutidos os resultados da pesquisa.

\section{RESULTADOS E DISCUSSÃO}

Neste tópico será explanado acerca dos trabalhos analisados, 10 artigos e 1 tese, e suas contribuições para a pesquisa na área abordada. A Figura 8 os divide em seus respectivos grupos. 


\section{$17^{\circ}$ ERGODESIGN \& USIHC 2019}

PUC-Rio, 11 a 13 de dezembro

Rio de Janeiro, RJ, Brasil $17^{\circ}$ Ergodesign - Congresso Internacional de Ergonomia e Usabilidade de Interfaces Humano Tecnológica: Produto, Informações Ambientes Construídos e Transporte

$17^{\circ}$ USIHC - Congresso Internacional de Ergonomia e Usabilidade de Interfaces Humano Computador

Figura 8. Título dos trabalhos analisados divididos em seus respectivos grupos

\begin{tabular}{|c|c|c|}
\hline Grupo 1 - 4 artigos & Grupo 2 - 2 artigos & Grupo 3 - 4 artigos e 1 tese \\
\hline $\begin{array}{l}\text { Riesgo ergonómico en empresas } \\
\text { artesanales del sector de la manufactura, } \\
\text { Santander. Colombia Ergonomic risk craft } \\
\text { enterprises manufacturing sector, } \\
\text { Santander. Colombia }\end{array}$ & $\begin{array}{l}\text { Delivering meat carcasses/cuts to } \\
\text { craft-butcher shops: An investigation of } \\
\text { work characteristics and manual handling } \\
\text { hazards }\end{array}$ & $\begin{array}{l}\text { Material Inheritances: How Place, } \\
\text { Materiality, and Labor Process Underpin } \\
\text { the Path-dependent Evolution of } \\
\text { Contemporary Craft Production }\end{array}$ \\
\hline $\begin{array}{l}\text { Ergonomic Redesign of a Traditional } \\
\text { Jewelry-Polishing Workstation }\end{array}$ & $\begin{array}{l}\text { Occupational traumatic injuries among } \\
\text { offshore seafood processors in Alaska, } \\
2010-2015\end{array}$ & $\begin{array}{l}\text { Crafting communities of practice: the } \\
\text { relationship between making and learning }\end{array}$ \\
\hline $\begin{array}{l}\text { An experimental study to evaluate } \\
\text { musculoskeletal disorders and postural } \\
\text { stress of female craftworkers adopting } \\
\text { different sitting postures }\end{array}$ & & $\begin{array}{l}\text { Research on the Application of Traditional } \\
\text { Embroidery Technology in Modern Jewelry } \\
\text { Creation }\end{array}$ \\
\hline \multirow[t]{2}{*}{$\begin{array}{l}\text { Prevalência dos distúrbios } \\
\text { musculoesqueléticos nos membros } \\
\text { superiores e pescoço em pescadoras } \\
\text { artesanais/ marisqueiras em Saubara, } \\
\text { Bahia, Brasil. }\end{array}$} & & $\begin{array}{l}\text { Collaboration and exchange between } \\
\text { "Craftsman" and "Designer": Symbiosis } \\
\text { towards Product Innovation. }\end{array}$ \\
\hline & & $\begin{array}{l}\text { A Gestão de Design e o Modelo de } \\
\text { Intervenção de Design para Ambientes } \\
\text { Artesanais: Um estudo de caso sobre a } \\
\text { atuação do Laboratório de Design O } \\
\text { Imaginário/UFPE nas comunidades produ- } \\
\text { toras Artesanato Cana-Brava - Goiana, e } \\
\text { Centro de Artesanato Wilson de Quei }\end{array}$ \\
\hline
\end{tabular}

Fonte: autoras (2019)

Nota-se que os trabalhos que possuíram alguma análise ergonômica no processo de produção artesanal totalizaram 6 artigos, divididos nos grupos 1 e 2, modificando apenas o nível da relevância da pesquisa; já os trabalhos que não abordam a ergonomia, mas que mesmo assim foram analisados, devido a suas aprovações nas leituras pelo resumo, totalizaram 4 artigos e 1 tese.

Com base no método PRISMA utilizado, as informações dos trabalhos foram sistematizadas em uma planilha do Excel (Figura 9) com as seguintes categorias: no do trabalho, título do trabalho, autor(es), referência, resumo, grupo do trabalho, país de origem do trabalho, objeto de estudo, introdução, objetivos, metodologia, métodos e ferramentas, análise do trabalho, resultados e conclusão. 
Figura 9. Estrutura da Planilha Excel com base no método PRISMA

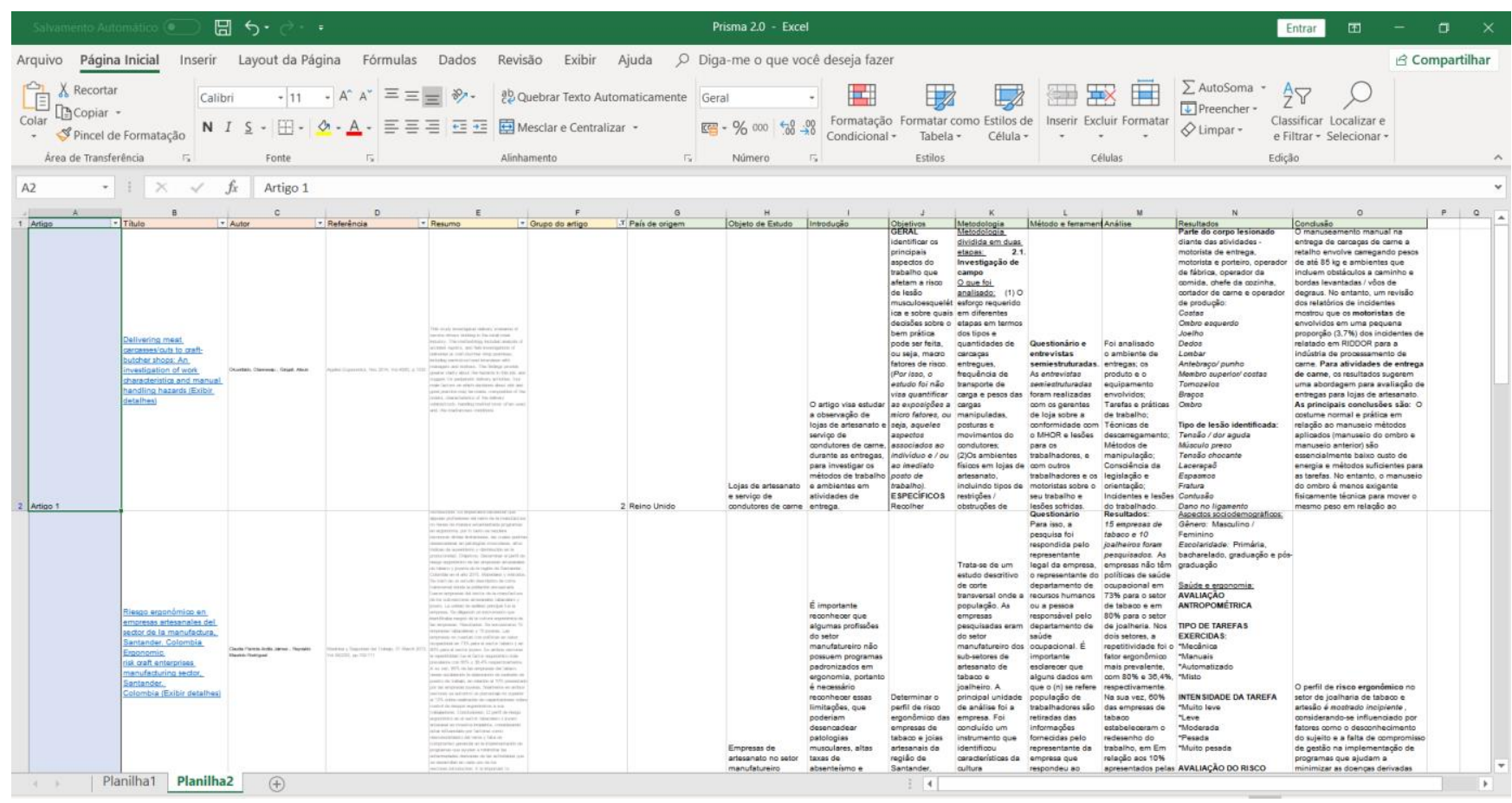

Fonte: autoras (2019)

Será apresentado a seguir um compilado dos dados levantados de cada grupo analisado.

\subsection{Grupo 1}

No grupo de maior relevância foram analisados 4 artigos que abordam a análise ergonômica em processos de produções artesanais, e eles foram sistematizados conforme as categorias listadas anteriormente. Logo compilando-os, temos:

- Riesgo ergonómico en empresas artesanales del sector de la manufactura, Santander. Colombia, por Claudia Patricia Ardila Jaimes e Reynaldo Mauricio Rodríguez, tem origem na Colômbia e seu objeto de estudo são empresas de artesanato no setor manufatureiro de Santander, Colômbia. O estudo visou determinar o perfil de risco ergonômico dessas empresas em 2010. Trata-se de um estudo descritivo de corte transversal e os métodos utilizados foram questionários sociodemográficos aplicados em 15 empresas de Tabaco e 10 Joalheiros. Concluindo o estudo, os autores apresentam que o perfil de risco ergonômico no setor artesanal de joalharia e tabaco é mostrado incipiente e influenciado por fatores como o desconhecimento do sujeito e a falta de compromisso de gestão na implementação de programas que ajudem a minimizar as doenças derivadas das atividades desenvolvidas nesses setores.

- Ergonomic Redesign of a Traditional Jewelry-Polishing Workstation, por Mauro, Charles L.; Fisher, Emily; Korpan, David; Medrano, P. Adrian, é um artigo estadunidense, e tem como objeto de estudo o posto de trabalho para a produção de joias. O objetivo da pesquisa é mitigar o movimento repetitivo e suas lesões provocadas, 
melhorando a saúde do trabalhador, sua produtividade, eficiência e sua carreira de polidor, reduzindo o tempo de treinamento necessário para novos polidores alcançarem o nível de critério profissional de desempenho de produção. Para tanto foi utilizada uma metodologia de pesquisa de estudo de caso e metodologia projetual de design centrado no usuário, cujas etapas constam avaliações ergonômicas para análise de faixas posturais. Mauro, et al (2015) estabeleceram um método de polimento e estação de trabalho que atende as normas da ergonomia e produção, exigências dos polidores de joias, melhorando a sua qualidade de vida, extensão e duração de suas carreiras.

- An experimental study to evaluate musculoskeletal disorders and postural stress of female craftworkers adopting different sitting postures, por Maity, Payel ; De, Sujaya ; Pal, Amitava ; Dhara, Prakash C., é um artigo de origem indiana e tem como objeto de estudo a postura de mulheres artesãs da Índia do estado de Bengala Ocidental. O objetivo foi o de avaliar os distúrbios musculoesqueléticos (DME) e o estresse postural dessas mulheres. A metodologia foi dividida em diversas fases de análises: Seleção do local; Avaliação do desconforto de parte do corpo: ângulo da articulação do ombro, ângulo da articulação do cotovelo, ângulo da articulação do pulso, ângulo da articulação do quadril e Ângulo da articulação do joelho; Estudo EMG (Eletromiografia); Seleção muscular; Análise do valor EMG e Análise estatística; Foram várias as ferramentas e métodos aplicados: (1) Aplicação do questionário nórdico modificado - durante a adoção das seguintes posturas: sentado no chão com as pernas dobradas, agachado e sentado no chão com as pernas esticadas; (2) Mapeamento de dor de Wilson e Corlett nas três posturas; (3) Os diferentes ângulos da articulação do corpo foram medidos com o goniômetro (Lafayette, EUA) - Medição dos ombros, cotovelos, pulso, quadril e joelho; (4) Estudo EMG - sistema de BIOPAC (EUA); E (5) Método Estatístico.

- Prevalência dos distúrbios musculoesqueléticos nos membros superiores e pescoço em pescadoras artesanais/marisqueiras em Saubara, Bahia, Brasil, por Falcao, Ila Rocha ; Couto, Maria Carolina Barreto Moreira ; Lima, Veronica Maria Cadena ; Pena, Paulo Gilvane Lopes ; Andrade, Lilian Lessa ; Muller, Juliana Dos Santos ; Alves, Ivone Batista ; Viana, Wendel Da Silva ; Rego, Rita de Cassia Franco, é uma pesquisa que ocorreu no Brasil, no estado da Bahia e possui como objeto de estudo uma comunidade de pescadores artesanais/marisqueiras de Saubara. $O$ artigo visa identificar demandas ergonômicas nos postos de trabalho dessas marisqueiras, que compõe um projeto mais amplo intitulado: Saúde, Ambiente e Sustentabilidade de trabalhadores da pesca artesanal. Para sua realização utilizou-se a pesquisa bibliográfica e epidemiológica. A pesquisa bibliográfica, realizada na Base de Dados Pubmed até fevereiro de 2014, objetivou localizar toda a bibliografia de âmbito nacional e internacional já tornada pública, em relação ao DME em membros superiores e pescoço, já o estudo epidemiológico de corte transversal foi realizado com 209 pescadoras artesanais/marisqueiras do município de Saubara, Bahia. A versão brasileira do Job Content Questionnaire (JCQ) e do Nordic Musculoskeletal Questionnaire (NMQ) foram utilizadas junto a um questionário contendo as demandas físicas adaptadas para o trabalho das marisqueiras a fim de levantar dados estatísticos.

Desse modo verificou-se que os artigos abordados se apresentam bem distribuídos geograficamente, além de que as metodologias, métodos e ferramentas utilizados foram bem vastos, tais como metodologia bibliográfica, de estudo de caso, métodos de análise ergonômica para faixas posturais, método estatístico, ferramenta de questionário, entre outros. No entanto, 


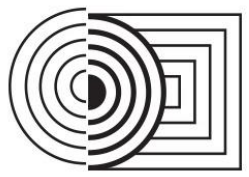

\section{$17^{\circ}$ ERGODESIGN \& USIHC 2019 \\ PUC-Rio, 11 a 13 de dezembro \\ Rio de Janeiro, RJ, Brasil}

$17^{\circ}$ Ergodesign - Congresso Internacional de Ergonomia e Usabilidade de Interfaces Humano Tecnológica: Produto, Informações Ambientes Construídos e Transporte

$17^{\circ}$ USIHC - Congresso Internacional de Ergonomia e Usabilidade

de Interfaces Humano Computador

notou-se uma semelhança maior na estrutura entre os dois últimos artigos, devido as avaliações posturais no posto de trabalho das artesãs da Índia e marisqueiras do Brasil, ambos realizaram análises ergonômicas posturais mais rebuscadas.

\subsection{Grupo 2}

Nesse grupo a relevância dos trabalhos foi considerada moderada, uma vez que apesar de serem realizadas análises ergonômicas nos processos de produção artesanais, as atividades realizadas em si não são relevantes para o estudo, tendo em vista a definição de artesanato descrita no tópico 2 deste presente artigo. Têm-se que os trabalhos analisados foram 2 artigos:

- Delivering meat carcasses/cuts to craft-butcher shops: An investigation of work characteristics and manual handling hazards, por Okunribido, Olanrewaju; Gingell, Alison, publicado no ano de 2014, no Reino Unido, aborda lojas de artesanato e serviço de condutores de carne. O artigo visa estudar a observação dessas lojas artesanais e serviço de condutores de carne, durante as entregas, para investigar os métodos de trabalho e ambientes em atividades de entrega. Sendo a metodologia dividida em (1) Investigação de campo, avaliando as atividades realizadas e os ambientes através da aplicação de questionários e entrevistas semiestruturadas, e (2) Análise de acidentes, que ocorreu avaliando o banco de dados do Sistema de Informações Operacionais Corporativas de HSE (COIN) e pesquisando por relatórios, feitos sob o Relatório de Regime de Doenças e Perigosas de Lesões de 1995 (RIDDOR 1995), de acidentes e incidentes de movimentação manual no indústria de carne. A busca foi restrita ao período de quatro anos de 2005 a 2008. Foi analisado o ambiente de entregas; os produtos e os equipamentos envolvidos; Tarefas, técnicas e práticas de trabalho; Métodos de manipulação; Consciência da legislação e orientação; Incidentes e lesões do trabalhado, a fim de identificar as partes dos corpos lesionadas e os tipos de lesão correspondente.

- Occupational traumatic injuries among offshore seafood processors in Alaska, 2010-2015, por Syron, Laura N.; Lucas, Devin L. ; Bovbjerg, Viktor E.; Case, Samantha; Kincl, Laurel, estuda processadores de frutos do mar no Alasca, Estados Unidos, e objetiva descrever padrões de lesões traumáticas entre processadores de frutos do mar em alto mar, bem como identificar riscos modificáveis. Syron et al (2018) coloca que as lesões mais frequentes foram: por natureza de lesão: entorse, estiramento, ruptura, contusões e fraturas; por parte do corpo afetada, membros superiores e tronco; por evento / exposição resultando em lesão: contato com objetos e equipamentos, esforço excessivo e reação corporal; e por fonte de lesão: equipamentos de processamento e máquina. Os autores ainda concluem que profissionais de segurança e pesquisadores podem usar as descobertas do estudo para informar futuros esforços de intervenção nessa indústria.

Logo, como observado nos estudos apresentados, os mesmos apesar de possuírem análises ergonômicas de processos de produção, os processos apresentados não são caracterizados como artesanais, manufatureiros e em série, com o objetivo de garantir o sustento do artesão e de sua família.

\subsection{Grupo 3}


Por não conter análise ergonômica, o grupo 3 é o grupo de menor relevância, visando somente o conteúdo dos artigos com relação ao processo de produção artesanal. Esse grupo contém 4 artigos e 1 tese, e as metodologias utilizadas foram: metodologia de pesquisa exploratória, bibliográfica, em um dos artigos e na tese, estudo de caso. Os métodos e ferramentas, quando utilizados, corresponderam ao método bibliográfico, analítico e dialético. Dividindo a análise entre os artigos e a tese, temos:

\subsubsection{Artigos}

- Material Inheritances: How Place, Materiality, and Labor Process Underpin the Path-dependent Evolution of Contemporary Craft Production, por Gibson, Chris, 2015, Estados Unidos, que analisa um caso especialmente vívido e explorado: um cluster de oficinas de bootmaking cowboy em El Paso, Texas, Estados Unidos, e busca destacar a materialidade e sua evolução histórica na análise da produção artesanal contemporânea.

- Crafting communities of practice: the relationship between making and learning, por Gibson, Miriam, 2018, Nova Zelândia, que estuda a percepção cambiante da atividade artesanal e seu processo de aprendizagem, visando estabelecer um panorama do processo de ensino-aprendizagem artesanal.

- Research on the Application of Traditional Embroidery Technology in Modern Jewelry Creation, por Min Li; Xiaoyan Zhang, 2017, Xangai, China, que aborda o trabalho de joias de bordar nas tradicionais cultura e técnica de bordados chineses, objetivando de modo geral estudar o método teórico de combinar a tecnologia de bordado tradicional com a moderna de joias.

- Collaboration and exchange between "Craftsman" and "Designer": Symbiosis towards Product Innovation, por Temeltaş, Handan, 2017, Istambul, Turquia. O artigo apresentou casos de desenvolvimento de novos produtos que artesãos e designers colaboram na indústria, e teve como objetivo definir características do artesanato que ajudaram a criar um possível ambiente de inovação.

\subsubsection{Tese}

- A Gestão de Design e o Modelo de Intervenção de Design para Ambientes Artesanais: Um estudo de caso sobre a atuação do Laboratório de Design 0 Imaginário/UFPE nas comunidades produtoras Artesanato Cana-Brava - Goiana, e Centro de Artesanato Wilson Wilson de Queiroz Campos Júnior - Cabo de Santo Agostinho, Pernambuco, por Ana Maria Queiroz de Andrade, 2015, Recife, Brasil. Estudo de caso, no municipio do Cabo em Pernambuco, que objetiva "propor um modelo de gestão para atuação de design no ambiente artesanal, especificamente para comunidades produtoras de artesanato, a partir da análise de modelos de gestão de design utilizados no ambiente industrial e do modelo de intervenção do Laboratório de Design O Imaginário e experiências vivenciadas nas comunidades artesãs Artesanato Cana Brava - Goiana, e Centro de Artesanato Wilson de Queiroz Campos Júnior. O estudo critica os modelos de gestão de design e suas atuações no artesanato e sugere adaptações que utilizam a visão empreendedora e de negócio nas propostas de intervenções artesanais.

Desse modo, têm-se que os artigos e a tese possuem abordagens distintas, como a análise do processo de produção artesanal contemporânea, o processo de aprendizagem na produção artesanal, a combinação entre artesanato, modernidade, tecnologia e inovação, e inserção de 


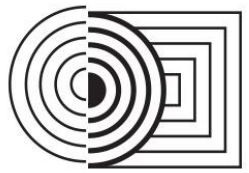

\section{$17^{\circ}$ ERGODESIGN \& USIHC 2019 \\ PUC-Rio, 11 a 13 de dezembro \\ Rio de Janeiro, RJ, Brasil}

$17^{\circ}$ Ergodesign - Congresso Internacional de Ergonomia e Usabilidade de Interfaces Humano Tecnológica: Produto, Informações Ambientes Construídos e Transporte

$17^{\circ}$ USIHC - Congresso Internacional de Ergonomia e Usabilidade

de Interfaces Humano Computador

intervenções de design no artesanato visando o empreendedorismo e a melhoria nos negócios, sendo que, exceto a tese, todos são de cunho teórico.

\section{CONSIDERAÇÕES FINAIS}

Nesta revisão, pode ser observada a diversidade geográfica da utilização de metodologias de Revisão Sistemática de Literatura. O estudo abrangeu os países: Colômbia, Estados Unidos, Índia, Brasil, China, Turquia, Nova Zelândia e Reino Unido. Além de que as metodologias, métodos e ferramentas utilizados foram bem vastos. A maioria das pesquisas mostraram a ocorrência do uso de metodologia bibliográfica, de estudo de caso, métodos de análise ergonômica, métodos estatísticos, aplicação de ferramentas ergonômicas e questionário para obtenção e análise dos dados.

A distribuição dos artigos nos grupos 1,2 e 3, pode relacionar as temáticas dos artigos analisados e as suas contribuições para a pesquisa, e mesmo os trabalhos que não apresentaram a ergonomia no seu contexto, podem contribuir para a pesquisa através de análises dos processos de produção artesanais e suas correlações com tecnologias contemporâneas. E fez-se comprovada através da revisão sistemática a ausência de estudos de análise ergonômica no processo de produção artesanal, mostrando que nesse campo há muito para ser explorado.

\section{REFERÊNCIAS}

ABERGO (Associação Brasileira de Ergonomia). O que é Ergonomia? Disponível em: $<$ http://www.abergo.org.br/>. Acesso em: 07 de novembro de 2018.

ANDRADE, A. M. Q. A Gestão de Design e o Modelo de Intervenção de Design para Ambientes Artesanais: Um estudo de caso sobre a atuação do Laboratório de Design 0 Imaginário/UFPE nas comunidades produtoras Artesanato Cana-Brava - Goiana, e Centro de Artesanato Wilson de Queiroz Campos Júnior - Cabo de Santo Agostinho, Pernambuco. 2015. Tese (Doutorado em Design) - Departamento de Design, Universidade Federal de Pernambuco, Recife. 395f.

ARDILA JAIMES, C. P. \& RODRíGUEZ, R. M. Riesgo ergonómico en empresas artesanales del sector de la manufactura, Santander. Colombia. Med Segur Trab (Internet), [s. I.], v. 59, n. 230, p. 102-111, 2013.

CORDEIRO, Alexander Magno et al. Revisão sistemática: uma revisão narrativa. Revista do Colégio Brasileiro de Cirurgiões, [s. I.], v. 34, n. 6, p. 428-431, 2007. Disponível em: $<$ http://www.scielo.br/scielo.php?script=sci_arttext\&pid=S0100-

$69912007000600012 \&$ Ing =pt\&tlng=pt>

DUARTE, A. Y. S. et al. O conhecimento tradicional e o desenvolvimento de produtos artesanais no campo do design. In: Anais do Interfaces Científicas: exatas e tecnológicas na Região Nordeste. Aracaju, 2015.

GIBSON, Chris. Material inheritances: How place, materiality, and labor process underpin the Path-Dependent evolution of contemporary craft production. Economic Geography, [s. I.], v. 92 , n. 1, p. 61-86, 2016.

GIBSON, Miriam. Crafting communities of practice: the relationship between making and learning. International Journal of Technology and Design Education, [s. I.], p. 1-11, 2018. Disponível em: <https://doi.org/10.1007/s10798-017-9430-3>.

LI, Min; ZHANG, Xiaoyan. Technology in Modern Jewelry Creation. [s. I.], V. 06, n. 10, p. 7 - 11, 2017. 
MAITY, Payel et al. An experimental study to evaluate musculoskeletal disorders and portural stress of female craftworkers adopting different sitting postures. International Journal of Occupational Safety and Ergonomics, [s. I.], v. 22, n. 2, p. $257-266,2016$.

MAURO, Charles L. et al. Ergonomic redesign of a traditional jewelry-polishing workstation. Ergonomics is Design, [s. I.], v. 23, n. 1, p. $4-12,2015$.

OKUNRIBIDO, Olanrewaju O.; GINGELL, Alisson. Delivering meat carcasses/cuts to craft-butcher shop: An investigation of work characteristics and manual handling hazards. Applied Ergonomics, [s. I.], v. 45, n. 6, p. 1530-1539, 2014. Disponível em: <http://dx.doi.org/10.1016/j.apergo.2014.04.016>.

TEMELTAS, Handas. Collaboration and exchange between "Craftsman" and "Designer": Symbiosis toward Product Innovation. The Design Journal, [s. I.], v. 20, n. sup1, p. S3713-S3723, 2017. Disponível em: <https://www.tandfonline.com/doi/full/10.1080/14606925.2017.1352876>.

SYRON, Laura N. et al. Occupational traumatic injuries among offshore seafood processors in Alaska, 2010-2015. Journal of Safety Research, [s. I.], v. 66, p. 169-178, 2018. Disponível em: <https://doi.org/10.1016/j.jsr.2018.07.008>

BRASIL, MINISTÉRIO DA SAÚDE, SECRETARIA DE CIÊNCIA, Técnologia e Insumos Estratégicos. Departamento de Ciiência e Tecnologia. S Me. [s.l: s.n.]. 